\title{
SEDs of massive protostars
}

\author{
Christopher F. McKee ${ }^{1}$ and Sukanya Chakrabarti ${ }^{2}$ \\ ${ }^{1}$ Departments of Physics and Astronomy, University of California, Berkeley CA 94720 USA \\ email: cmckee@astro.berkeley.edu \\ ${ }^{2}$ Department of Physics, Univerisity of California, Berkeley CA 94720 USA \\ email: sukanya@astro.berkeley.edu
}

\begin{abstract}
Massive protostars are generally enshrouded in dust, so that most of their radiation emerges in the far infrared. For protostars embedded in opaque, spherical cores, the spectral energy distribution (SED) is determined by two distance-independent parameters, the luminosityto-mass ratio, $L / M_{c}$, and the surface density of the core, $\Sigma=M_{c} /\left(\pi R_{c}^{2}\right)$, where $R_{c}$ is the radius of the core. Chakrabarti \& McKee (2005a) have derived an approximate analytic expression for the SED that agrees well with numerical results. It is generally not possible to infer the power-law of the density from the SED of a massive protostar. Masses and accretion rates are inferred for several well-studied sources.
\end{abstract}

\section{Introduction}

The far-infrared spectral energy distribution (SED) is a vital implement in understanding the physical conditions in the environments of massive protostars (Osorio, Lizano, \& D'Alessio 1999, henceforth OLD). Chakrabarti \& McKee (2005a, henceforth CM I) developed self-consistent analytic radiative transfer solutions for the spectra of unresolved, homogeneous, spherically symmetric, centrally heated, dusty sources. Chakrabarti \& McKee (2005b, henceforth CM II) applied this methodology to sources ranging from lowmass protostars to dusty galaxies. Here, we apply this methodology to massive protostars in order to infer the properties of the protostars and the molecular cores out of which they are forming.

Inferring the properties of massive protostars is of particular interest today as there is no clear consensus on the formation mechanism of massive stars. The conventional view is that massive stars form via gravitational collapse (e.g, Wolfire \& Cassinelli 1987; OLD). The Turbulent Core Model (McKee \& Tan 2002; 2003, henceforth MT03) put this idea on a firmer footing by incorporating the effects of the supersonic turbulence and the high pressures observed in massive star-forming regions (Plume et al 1997); it predicts formation times of $\sim 10^{5} \mathrm{yr}$. However, concerns about the difficulty in forming massive stars in the face of the enormous radiation pressure associated with massive stars led to the development of an alternative theory, that massive stars are formed by the coalescence of low-mass stars (Bonnell, Bate, \& Zinnecker 1998, Bonnell, Vine, \& Bate 2004, Bally \& Zinnecker 2005). It is therefore important to attempt to distinguish these theories through observation.

\section{Analytic SEDs of Dusty Sources}

In CM I, we formulated an analytic solution for the far-IR SEDs of spherically symmetric, homogeneous dusty sources with a central source of radiation. We extensively tested the accuracy of our analytic solution over a parameter space that spans low-mass protostars to ULIRGs by comparing it against the numerical scheme, DUSTY (Ivezic 
\& Elitzur 1997) and found excellent agreement with the numerical results. We assumed that the sources emitted most of their radiation at wavelengths longer than $30 \mu \mathrm{m}$; under these conditions, the far-IR SED is approximately independent of the temperature of the source of radiation and of the properties of the dust destruction front. At these wavelengths, scattering is negligible.

We assumed a power-law density variation within the envelope, $\rho(r) \propto r^{-k_{\rho}}$. We adopted a dust opacity curve, $\kappa_{\nu}$, from Weingartner \& Draine (2001), with an opacity normalization appropriate for water ice mantles. For a given density variation in the envelope and dust opacity curve, the emergent spectrum depends on three quantities the mass of the core, $M_{c}=M_{c \text {, dust }} / Z_{\text {dust }}$; the luminosity, $L$; and the radius of the envelope, $R_{c}$. The shape of the SED cannot depend on the distance to the source, and therefore can be specified by two distance-independent parameters, the luminosity to mass ratio, $L / M_{c}$, and the surface density, $\Sigma \equiv M_{c} / \pi R_{c}^{2}$.

We defined characteristic parameters, $R_{\mathrm{ch}}$ and $T_{\mathrm{ch}}$, that correspond to the Rosseland photosphere and photospheric temperature respectively, such that

$$
L \equiv 4 \pi \tilde{L} R_{\mathrm{ch}}^{2} \sigma T_{\mathrm{ch}}^{4} .
$$

Here, $\tilde{L}$ is a number of order unity that allows for better accuracy, particularly for extended atmospheres, which have $\tilde{L}$ somewhat greater than unity. The characteristic parameters are determined by requiring that the characteristic optical depth at a frequency $\nu_{\mathrm{ch}} \equiv k T_{\mathrm{ch}} / h$ equal unity,

$$
\tau_{\mathrm{ch}}=\kappa_{\mathrm{ch}} \int_{1}^{R_{c} \rightarrow \infty} \rho(\tilde{r}) d \tilde{r}=\frac{\kappa_{\nu_{\mathrm{ch}}} \rho\left(R_{\mathrm{ch}}\right) R_{\mathrm{ch}}}{k_{\rho}-1}=1,
$$

where we have defined the characteristic optical depth under the assumption that the outer radius of the envelope is at infinity. We assume that the characteristic frequency, $\nu_{\mathrm{ch}}$, is within the frequency range where the opacity per unit mass is approximately a power law,

$$
\kappa_{\nu}=\kappa_{\nu_{0}}\left(\nu / \nu_{0}\right)^{\beta} \quad(30 \mu \mathrm{m}<\lambda<1 \mathrm{~mm}) ;
$$

we take $\beta=2$ in numerical evaluations. By solving equations (2.1) and (2.2), one can obtain the relations between the source parameters, $L / M_{c}$ and $\Sigma$, and the SED variables, $\tilde{R}_{c}$ and $T_{\text {ch }}$ that govern the shape of the SED (CM I).

Our analytic expression for the emergent spectrum is formulated within a "shell" model for radiative transfer - the emission at each frequency comes from a shell of thickness $\Delta r_{m}(\nu)$ centered at a radius $r_{m}(\nu)$, with a source function $\left(2 h \nu^{3} / c^{2}\right) \exp \left[-h \nu / k T\left(\tilde{r}_{m}\right)\right]$ located at an optical depth $\tau_{\nu}\left(\tilde{r}_{m}\right)$ :

$$
L_{\nu}=4 \pi R_{\mathrm{ch}}^{2} 4 \pi\left(\frac{2 h \nu_{c h}^{3}}{c^{2}}\right) \tilde{\kappa}_{\nu} \tilde{\nu}^{3}\left(k_{\rho}-1\right) \tilde{r}_{m}^{2-k_{\rho}} \Delta \tilde{r}_{m} \exp \left[-\frac{h \nu}{k T\left(\tilde{r}_{m}\right)}-\tau_{\nu}\left(\tilde{r}_{m}\right)\right],
$$

where the optical depth $\tau_{\nu}$ from $r$ to the surface of the cloud is

$$
\tau_{\nu}=\tilde{\kappa}_{\nu}\left(\tilde{r}^{-k_{\rho}+1}-\tilde{R}_{c}^{-k_{\rho}+1}\right) .
$$

The far-IR emission can be accurately represented with the adoption of a power law for the temperature profile,

$$
T=T_{\mathrm{ch}}\left(\frac{r}{R_{\mathrm{ch}}}\right)^{-k_{T}}=T_{\mathrm{ch}} \tilde{r}^{-k_{T}},
$$

The slope of the temperature profile, $k_{T}$, is determined by imposing the self-consistency 
condition that the input luminosity exactly equal the emergent luminosity. For extended envelopes, $k_{T} \rightarrow \frac{1}{3}$ (Adams 1991), but it becomes larger for more opaque envelopes. CM I gives a numerical expression for $k_{T}$ as a function of $\tilde{R}_{c}$ and $k_{\rho}$.

We showed that source spectra are characterized by three frequency regimes: (1) Low frequencies are in the Rayleigh-Jeans limit $(h \nu \ll k T)$ and are opticallly thin. Since the optically thin flux is proportional to the mass, the emission radius $\tilde{r}_{m}$ is comparable to $\tilde{R}_{c}$. (2) Intermediate frequencies are characterized by $h \nu \sim k T\left(\tilde{r}_{m}\right)$, so that higher frequencies probe deeper into the envelope, where it is hotter. (3) High frequencies emanate from a location in the shell that is due to a tug-of-war between the temperature gradient and the intervening optical depth that has to be traversed. In CM I we were able to calculate $\tilde{r}_{m}(\nu)$ and $\Delta \tilde{r}_{m}(\nu)$ for each regime and then smoothly join them so as to obtain an explicit expression for $L_{\nu}$.

\section{Inferring the Properties of Protostellar Envelopes}

Since massive protostars preferentially form in clusters, the high frequency emission may be due to sources that are not associated with the core itself, or it may be escaping from the inner part of the core due to an inhomogenous distribution of dust within the envelope. Therefore, in fitting for the SED parameters, we focus on emission at long wavelengths $(\lambda \geqslant 60 \mu \mathrm{m})$, which is less likely to have been affected by sources external to the core. In fitting the SED, we consider single dish data since interferometers may resolve out the large-scale emission.

We model IRAS 18454-0136 from the sample of high-mass protostellar objects in the survey by Sridharan and Beuther (Sridharan et al. 2002; Beuther et al. 2002). We give in Table 1 the distance - independent parameters for this source only, i.e., $L / M_{c}$ and $\Sigma$, as the distance ambiguity to this source has not been resolved. The source IRAS $21307+5049$ is a well-studied example of a massive protostar (Fontani et al. 2004, Molinari et al. 2002). Located at a kinematic distance of $3.2 \mathrm{kpc}$, the massive core is surrounded by a cluster of more evolved stars that are seen clearly at $2 \mu \mathrm{m}$ (Fontani et al. 2004). This structure, i.e., a massive core located close to the center of the clump surrounded by a cluster of lower-mass stars seen in the near-infrared, has been observationally detected in other regions of massive star formation as well.

Tables 1 and 2 summarize the results of our analysis of the SEDs of massive protostars. In addition to IRAS 18454-0136 and IRAS 21307+5049, we also present results for an infrared dark cloud (IRDC), using data kindly provided by Jackson \& Rathborne from the recent BU-FCRAO survey. IRDCs were first seen as dark patches in the ISOCAM mid-IR images (Perault et al. 1996). Since they are optically thick at $15 \mu \mathrm{m}$, they must have $\Sigma \gtrsim 0.2 \mathrm{~g} \mathrm{~cm}^{-2}$. They were later seen as dark spots in the MSX surveys (Egan et al. 1999) at $8 \mu \mathrm{m}$ and were most recently detected by the Spitzer Space Telescope's MIPS at $24 \mu \mathrm{m}$ (Jackson \& Rathborne, private communication), and mapped in ${ }^{13} \mathrm{CO}$ and millimeter continuum in the BU-FCRAO survey (Rathborne et al. 2003).

\section{Collapsing Protostellar Envelopes}

Within the context of inside-out collapse models (Shu 1977), collapsing protostellar cores go through three stages of evolution: 1) the expansion wave, at $R_{\mathrm{ew}}$, is inside the photosphere; 2 ) the expansion wave is beyond the photosphere, so that the photosphere is in the free fall region, but still within the core $\left(R_{\mathrm{ch}}<R_{\mathrm{ew}}<R_{c}\right)$; and 3$)$ the expansion wave has moved beyond the core radius $\left(R_{\text {ew }}>R_{c}\right)$. Let $f_{\text {ew }}$ be the fraction of the mass inside the expansion wave that has accreted onto the star; McLaughlin \& Pudritz (1997) 
show that $f_{\text {ew }} \simeq(1 / 2,1 / 6,1 / 33)$ for $k_{\rho}=(2,1.5,1)$. Well inside the expansion wave radius, the density profile approaches $\rho \propto r^{-3 / 2}$ regardless of the shape of the initial density profile; as a result, sources near the end of Stage 2 or in Stage 3 are likely to be characterized by $k_{\rho}=\frac{3}{2}$.

Our spherical model requires that the emission from a protostellar disk not dominate the spectrum. Since the flow becomes very non-spherical inside the disk radius, $R_{d}$, we require $R_{d}<R_{\mathrm{ch}}$. The magnitude of the disk radius depends on the rotation of the cloud, which Goodman et al. (1993) characterized in terms of the parameter $\beta \equiv \Omega^{2} R^{3} /(3 G M)$; they found that typically $\beta \simeq 0.02$. An element of mass contracting at constant angular momentum has $\beta \propto 1 / R$; as a result, the disk radius at any time is related to the initial radius of a mass element $R(M)$ by $R_{d}=3 \beta R(M)=3 \beta\left(M / M_{c}\right)^{1 /\left(3-k_{\rho}\right)} R_{c}$. We assume that the rotational velocity $\Omega R$ is proportional to the velocity dispersion $\sigma$ and that the cloud is initially in hydrostatic equilibrium; then $\beta$ is independent of $M$. If the core has a value of $\beta$ similar to that found by Goodman et al. (which is also what is found in numerical simulations of turbulent flows-Burkert \& Bodenheimer 2000), then $R_{d}=0.06\left(m_{*} / m_{* f}\right)^{2 / 3} R_{c}$, where we have set $k_{\rho}=\frac{3}{2}$.

We derive accretion rates for massive protostars within the context of the Turbulent Core Model presented in MT03. As in MT03, we assume that cores and clumps are part of a turbulent, self-similar structure, with a mean power law index of $3 / 2$. In that case, the accretion rate may be expressed as:

$$
\dot{m}=4.6 \times 10^{-4}\left(\frac{m_{\star f}}{30 M_{\odot}}\right)^{3 / 4} \Sigma_{\text {clump }}^{3 / 4}\left(\frac{m_{\star}}{m_{\star f}}\right)^{0.5} M_{\odot} \mathrm{yr}^{-1}
$$

(MT03). To derive accretion rates within the context of the MT03 model, we first solve for $L / M_{c}$ and $\Sigma$, and for sources for which we know the distance, we solve for the luminosity, mass, and radius from the observed SED. We then use the model of protostellar evolution presented in MT03 to infer the stellar mass from the derived luminosity, and assume an average star formation efficiency $\epsilon=0.5$ to derive accretion rates using equation (4.1). We take $m_{\star f}=\epsilon M_{c}$. We solve for the location of the expansion wave radius and estimate the size of the accretion disk and whether or not it may influence the far-IR spectrum.

\section{SEDs \& Inferred Parameters}

From our fit to the observed SED for IRAS 18454-0136, we find that this source is compact, $\tilde{R}_{c} \sim 26$ (i.e., the core radius is a factor $\sim 20$ out from the Rosseland photosphere), and warm, $T_{\text {ch }} \sim 90 \mathrm{~K}$. This corresponds to an outer core temperature of $19 \mathrm{~K}$. We find a luminosity to mass ratio of $11 L_{\odot} / M_{\odot}$, and a surface density of $1.5 \mathrm{~g} \mathrm{~cm}^{-2}$. Beuther et al.'s (2002) $1.2 \mathrm{~mm}$ IRAM $30 \mathrm{~m}$ observations of this source show that this source appears to have a central dense core. However, their angular resolution of $11^{\prime \prime}$ corresponds to a length scale $\sim 0.5 \mathrm{pc}$ at a (far) distance of $11.9 \mathrm{kpc}$, much greater than the size of cores. It would be useful to map this region with a factor of ten higher resolution to ascertain whether this source is in fact dominated by an individual star (or binary).

We find that IRAS $21307+5049$ has a surface density $\Sigma=0.45 \mathrm{~g} \mathrm{~cm}^{-2}$, a core radius $R_{c}=0.12 \mathrm{pc}$, a core mass of $M_{C}=93 M_{\odot}$, and a luminosity $L=2.7 \times 10^{3} L_{\odot}$. Fontani et al. (2004) made a grey-body fit to these data and found $L \sim 4 \times 10^{3} L_{\odot}, T_{\text {dust }} \simeq 30 \mathrm{~K}$, and a mass of $90 M_{\odot}$. The OVRO map for this source indicates a compact core with a FWHM of 0.09 pc (Molinari et al. 2002), comparable to the radius we inferred from the SED alone; the observed temperature is close to our outer core temperature of $20 \mathrm{~K}$. As discussed in CMII, the inference of source parameters is still within our margin of error $(\sim 1.5)$ for massive protostars even when the density structure is not a pure power-law. 
Table 1. Source Parameters from SED

\begin{tabular}{lcccccc}
\hline Source & $L\left(L_{\odot}\right)$ & $M_{c}\left(M_{\odot}\right)$ & $R_{c}(\mathrm{pc})$ & $\Sigma\left(\mathrm{g} \mathrm{cm}^{-2}\right)$ & $\tilde{R}_{c}$ & $T_{\mathrm{ch}}$ \\
\hline I18454 & $L / M=11 L_{\odot} / M_{\odot}$ & - & - & $26 \pm 20$ & $87 \pm 11$ & \\
I21307 & $2.7 \times 10^{3}$ & 93 & 0.12 & 0.45 & $57 \pm 7$ & $119 \pm 5$ \\
IRDC43 & $6 \times 10^{3}-1.5 \times 10^{3}$ & $510-470$ & $0.53-0.15$ & $0.12-1.3$ & $45-350$ & $75-150$ \\
\hline
\end{tabular}

The major difference between our results and Fontani et al.'s is that we find that the temperature increases inward, reaching about $120 \mathrm{~K}$ at $r=0.002 \mathrm{pc}$. This value for the temperature is essential for us to match the 60 and $100 \mu \mathrm{m}$ fluxes. How is it possible for Fontani et al. to match the fluxes at a significantly lower temperature? The reason is that they assumed that the emission is optically thin, whereas in fact $\tau(60 \mu \mathrm{m})>4$; when this opacity is taken into account, a higher temperature is needed to match the observations. In any case, the temperature must rise inward if the observed luminosity is coming from a central source.

The Turbulent Core Model is predicated on the assumption that the core is in approximate virial equilibrium, so the virial parameter $\alpha \equiv 5 \sigma^{2} R / G M$ must be of order unity (Bertoldi \& McKee 1989). To compute the velocity dispersion from observation, it is important to measure the line width in a line that has negligible broadening from optical depth effects, and that tends to trace bound motions $\left(\mathrm{NH}_{3}\right)$ rather than outflows $\left(\mathrm{HCO}^{+}\right)$(Myers \& Goodman 1988). Using the linewidth of $\mathrm{H}^{13} \mathrm{CO}$ measured by Molinari et al. (2002), we find $\alpha_{\text {vir }}=1.5$, i.e., that the core is indeed in approximate virial equilibrium. We find that that expansion wave is at $0.06 \mathrm{pc}$, which places this source near the end of Stage 2.

Fontani et al. (2004) note that IRAS $21307+5049$ is quite similar in morphology to IRAS $23385+60533$, one of the earliest examples of a massive protostar (Molinari et al. 1998). However, recent Spitzer observations appear to show that the far-IR emission is not originating from the compact core (Stecklum et al., this conference). It would be valuable to observe IRAS $21307+5049$ with Spitzer as well in order to determine the validity of our assumption that the FIR emission arises from a core of dimension $\sim 0.002$ pc.

No far-IR data are available for the infrared dark cloud. As a result, $T_{\text {ch }}$ and $\tilde{R}_{c}$ are not well-constrained. In doing the fit, the $24 \mu \mathrm{m}$ flux was treated both as a data point and as an upper bound since, as discussed previously, it is quite possible that the $24 \mu \mathrm{m}$ data are affected by either clumping or other sources of luminosity. We give our inferred parameters for both these cases; for the case in which the $24 \mu \mathrm{m}$ flux is treated as an upper bound, we in fact took the minimum value consistent with the $\mathrm{mm}$ data. This case shows that without far-IR data, the inferred parameters will not be well-constrained. In particular, when we consider the $24 \mu \mathrm{m}$ point as a data point, we infer $\Sigma=0.12 \mathrm{~g} \mathrm{~cm}^{-2}$, while when taking the minimum possible value of the $24 \mu \mathrm{m}$ flux, we find $\Sigma=1.3 \mathrm{~g} \mathrm{~cm}^{-2}$. In either case, this source would be classified as being in Stage 2. This source has a lower outer core temperature than the other two sources, $T_{\mathrm{c}} \sim 14 \mathrm{~K}$; it would be worthwhile to measure the temperature in various tracers to confirm this.

\section{Acknowledgements}

The research of CFM is supported in part by the NSF through grant AST00-98365 and by the Miller Foundation for Basic Research. 
Table 2. Protostellar Parameters from SED

\begin{tabular}{lccccc}
\hline Source & $\dot{m}\left(M_{\odot} / y r\right)$ & $m_{\star}\left(M_{\odot}\right)$ & $R_{\text {ew }}(\mathrm{pc})$ & $R_{\text {disk }}(\mathrm{AU})$ & $t_{\text {age }}(\mathrm{yr})$ \\
\hline I18454 & - & - & - & - & - \\
I21307 & $1.3 \times 10^{-4}$ & 6 & 0.06 & 340 & $5 \times 10^{4}$ \\
IRDC43 & $7 \times 10^{-5}-4 \times 10^{-4}$ & 6 & $0.09-0.03$ & $540-160$ & $8 \times 10^{4}-1.4 \times 10^{4}$ \\
\hline
\end{tabular}
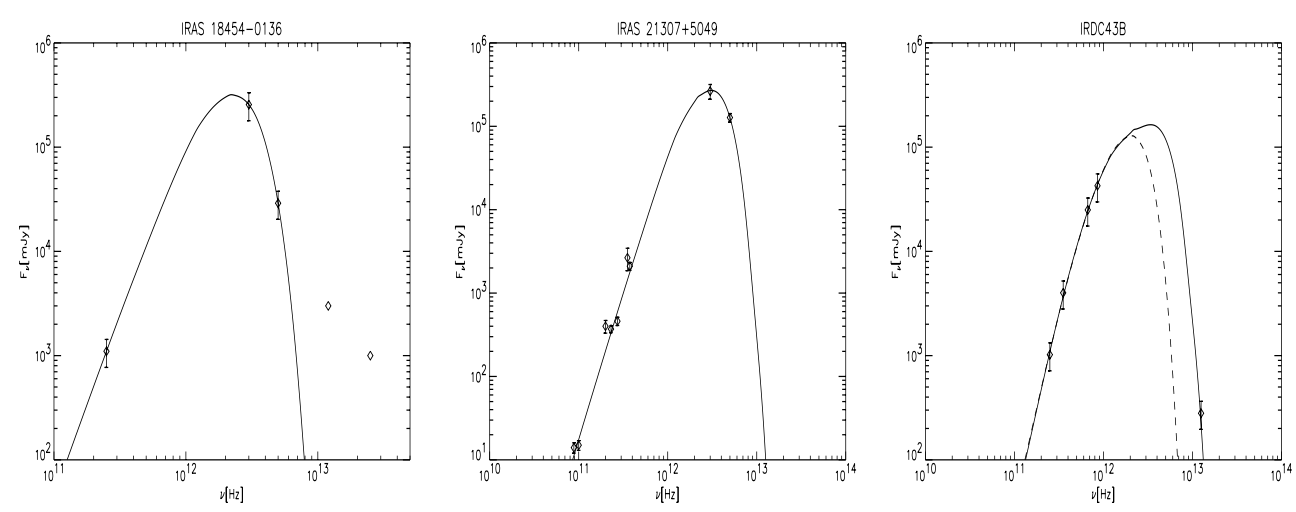

Figure 1. (a)-(c) best fit SEDs of (a) I18454, (b)I21307, (c) IRDC43, core B

\section{References}

Bally, J., \& Zinnecker, H. 2005, accepted by ApJ, astro-ph/0502485

Beuther, H., Schilke, P., et al. (2002), ApJ, 566, 945

Bonnell, I.A., Bate, M.R., \& Zinnecker, H. 1998, MNRAS, 298, 93

Bonnell, I.A., Vine, S.G., \& Bate, M.R. 2004, MNRAS, 349, 735

Burkert, A., \& Bodenheimer, P. 2000, ApJ, 543, 822-830

Chakrabarti, S., \& McKee, C.F. 2005a, accepted to ApJ

Chakrabarti, S., \& McKee, C.F. 2005b, in prep

Egan, M.P. et al. 1999, Universe as Seen By Iso conference proceedings, $671 \mathrm{E}$

Fontani, F., Cesaroni, R., et al. 2004, A\&A, 414, 209-315

Fontani, F., Cesaroni, R., et al. 2004, A\&A, 424, 179-195

Laughlin, G., \& Bodenheimer, P. 1994, ApJ, 436, 335-354

McKee, C.F., \& Tan, J.C. 2002, Nature, 416, 59

McKee, C.F., \& Tan, J.C. 2003, ApJ, 585, 850

McLaughlin, D.E., \& Pudritz, R.E. 1997, ApJ, 476, 750

Molinari, S. et al. 2002, ApJ, 570, 758

Myers, P., \& Goodman, A.A. 1988, ApJ, 327, L27

Osorio, M., Lizano, S., \& D'Alessio 1999, ApJ, 525, 808

Perault, M., et al. 1996, A\&A, 315, L165

Plume et al. 1997, ApJ, 476, 730

Rathborne, J.M., Simon, R. et al. 2004, ASPC, 322, 273

Sridharan, T.K., et al. 2002, ApJ, 566, 931

Weingartner, J.C., \& Draine, B.T. 2001, ApJ, 548, 296 ApJ, 548, 296

Wolfire, M., \& Casenelli, J. 1987, ApJ, 319, 850 